\title{
Extremal graph for intersecting odd cycles
}

\author{
Xinmin $\mathrm{Hou}^{a *} \quad \mathrm{Yu} \mathrm{Qiu}^{b} \quad$ Boyuan $\mathrm{Liu}^{b}$ \\ Key Laboratory of Wu Wen-Tsun Mathematics \\ Chinese Academy of Sciences \\ School of Mathematical Sciences \\ University of Science and Technology of China \\ Hefei, Anhui 230026, China. \\ ${ }^{a}$ xmhou@ustc.edu.cn, ${ }^{b}$ yuqiu@mail.ustc.edu.cn \\ ${ }^{c}$ lby 1055 mail.ustc.edu.cn
}

Submitted: Jan 8, 2016; Accepted: Apr 24, 2016; Published: May 13, 2016

Mathematics Subject Classifications: 05C35, 05C38

\begin{abstract}
An extremal graph for a graph $H$ on $n$ vertices is a graph on $n$ vertices with maximum number of edges that does not contain $H$ as a subgraph. Let $T_{n, r}$ be the Turán graph, which is the complete $r$-partite graph on $n$ vertices with part sizes that differ by at most one. The well-known Turán Theorem states that $T_{n, r}$ is the only extremal graph for complete graph $K_{r+1}$. Erdös et al. (1995) determined the extremal graphs for intersecting triangles and Chen et al. (2003) determined the maximum number of edges of the extremal graphs for intersecting cliques. In this paper, we determine the extremal graphs for intersecting odd cycles.
\end{abstract}

Keywords: extremal graph; Turán graph; intersecting odd cycle

\section{Introduction}

In this paper, all graphs considered are simple and finite. For a graph $G$ and a vertex $x \in V(G)$, the neighborhood of $x$ in $G$ is denoted by $N_{G}(x)$. Let $N_{G}[x]=\{x\} \cup N_{G}(x)$. The degree of $x$, denoted by $\operatorname{deg}_{G}(x)$, is $\left|N_{G}(x)\right|$. Let $\delta(G)$ and $\Delta(G)$ denote the minimum and maximum degrees of $G$, respectively. A matching $M$ in $G$ is a subgraph of $G$ with $\delta(M)=\Delta(M)=1$. The matching number of $G$, denoted by $\nu(G)$, is the maximum number of edges in a matching in $G$. Let $e(G)$ be the number of edges of $G$. For a graph $G$ and $S, T \subset V(G)$, let $e_{G}(S, T)$ be the number of edges $e=x y \in E(G)$ such that $x \in S$

\footnotetext{
*Supported by NNSF of China (No. 11271348) and the Fundamental Research Funds for the Central Universities.
} 
and $y \in T$, if $S=T$, we use $e_{G}(S)$ instead of $e_{G}(S, S)$; and we use $e_{G}(u, T)$ instead of $e_{G}(\{u\}, T)$ for convenience, the index $G$ will be omitted if no confusion from the context. For a subset $X \subseteq V(G)$ or $X \subseteq E(G)$, let $G[S]$ be the subgraph of $G$ induced by $X$, that is $G[X]=(X, E(X))$ if $X \subseteq V(G)$, or $G[X]=(V(X), X)$ if $X \subseteq E(G)$. A cycle of length $q$ is called a $q$-cycle.

Given two graphs $G$ and $H$, we say that $G$ is $H$-free if $G$ does not contain an $H$ as a subgraph. The Turán function, denoted by $\operatorname{ex}(n, H)$, is the largest possible number of edges of an $H$-free graph on $n$ vertices. That is,

$$
\operatorname{ex}(n, H)=\max \{e(G):|V(G)|=n, G \text { is } H \text {-free }\} .
$$

And for positive integers $n \geqslant r$, the Turán graph, denoted by $T_{n, r}$, is the complete $r$-partite graph on $n$ vertices with part sizes that differ by at most one (also called the complete balanced $r$-partite graph). The well-known Turán Theorem states that ex $\left(n, K_{r+1}\right)=$ $e\left(T_{n, r}\right)$ and $T_{n, r}$ is the only extremal graph for complete graph $K_{r+1}$.

A $k$-fan, denoted by $F_{k}$, is a graph on $2 k+1$ vertices consisting of $k$ triangles which intersect in exactly one common vertex. In 1995, Erdős et al. [4] gave the value of ex $\left(n, F_{k}\right)$ and determined the extremal graphs for $F_{k}$ as follows.

Theorem 1 ([4]). For $k \geqslant 1$ and $n \geqslant 50 k^{2}$,

$$
\operatorname{ex}\left(n, F_{k}\right)=e\left(T_{n, 2}\right)+g(k)
$$

where

$$
g(k)= \begin{cases}k^{2}-k & \text { if } k \text { is odd, } \\ k^{2}-\frac{3}{2} k & \text { if } k \text { is even } .\end{cases}
$$

Moreover, when $k$ is odd, the extremal graph must be a $T_{n, 2}$ with two vertex disjoint copies of $K_{k}$ embedding in one partite set. When $k$ is even, the extremal graph must be a $T_{n, 2}$ with a graph having $2 k-1$ vertices, $k^{2}-\frac{3}{2} k$ edges with maximum degree $k-1$ embedded in one partite set.

In 2003, Chen et al. [2] proved that $e x\left(n, F_{k, r}\right)=e\left(T_{n, r-1}\right)+g(k)$, where $F_{k, r}$ is a graph consisting of $k$ complete graphs of order $r$ which intersect in exactly one common vertex and $g(k)$ is the same as in Theorem 1. Recently, Glebov [5] and Liu [6] gave the extremal graphs for blow-ups of paths [5], cycles and a large class of trees [6] (A blow-up of a graph $G$ is a graph obtained from $G$ by replacing each edge of $G$ by a clique, two cliques share a common vertex if and only if their corresponding edges have a common end).

In this paper, motivated by the results in $[2,4,5,6]$, we generalize Theorem 1 in another way. For a positive integer $k$ and an odd integer $q$ with $q \geqslant 5$, let $C_{k, q}$ be the graph consisting of $k q$-cycles which intersect exactly in one common vertex, called the center of it. For $n \geqslant 4(k-1)^{2}$, let $\mathcal{F}_{n, k}$ be the family of graphs where each member is a Turán graph $T_{n, 2}$ with a complete bipartite graph $K_{k-1, k-1}$ embedded into one class. Our main result is as follows. 
Theorem 2. For an integer $k \geqslant 2$ and an odd integer $q \geqslant 5$, there exists $n_{1}(k, q) \in \mathbb{N}$ such that for all $n \geqslant n_{1}(k, q)$, we have

$$
\operatorname{ex}\left(n, C_{k, q}\right)=e\left(T_{n, 2}\right)+(k-1)^{2},
$$

and the only extremal graphs for $C_{k, q}$ are members of $\mathcal{F}_{n, k}$.

The remainder of the paper is arranged as follows. Section 2 gives some lemmas. Section 3 gives the proof of Theorem 2 .

\section{Lemmas}

The following two lemmas are useful to estimate the number of edges of a graph with restricted degrees and matching number.

Lemma 3 ( Chavátal [1]). For any graph $G$ with maximum degree $\Delta \geqslant 1$ and matching number $\nu \geqslant 1$, then $e(G) \leqslant f(\nu, \Delta)=\nu \Delta+\left\lfloor\frac{\Delta}{2}\right\rfloor\left\lfloor\frac{\nu}{\lceil\Delta / 2\rceil}\right\rfloor \leqslant \nu(\Delta+1)$.

The following stability result due to Erdös [3] and Simonovits [7] gives the rough structure of the extremal graphs for a graph $H$ with $\chi(H)=r \geqslant 3$ and $H \neq K_{r}$.

Lemma 4 ([3, 7]). Let $H$ be a graph with $\chi(H)=r \geqslant 3$ and $H \neq K_{r}$. Then, for every $\gamma>0$, there exists $\delta>0$ and $n_{0}=n_{0}(H, \gamma) \in \mathbb{N}$ such that the following holds. If $G$ is an $H$-free graph on $n \geqslant n_{0}$ vertices with $e(G) \geqslant \operatorname{ex}(n, H)-\delta n^{2}$, then there exists a partition of $V(G)=V_{1} \dot{\cup} \cdots \dot{U} V_{r-1}$ such that $\sum_{i=1}^{r-1} e\left(V_{i}\right)<\gamma n^{2}$.

The following is a simple observation.

Observation 5. Let $G$ be a graph with no isolated vertex. If $\Delta(G) \leqslant 2$, then

$$
\nu(G) \geqslant \frac{|V(G)|-\omega(G)}{2},
$$

where $\omega(G)$ is the number of components of $G$.

Proof. Since $\Delta(G) \leqslant 2$, each component of $G$ is a path or a cycle. Hence each component $C$ of $G$ has matching number at least $\frac{|V(C)|-1}{2}$. This implies the desired result.

Lemma 6. Let $G$ be a graph with no isolated vertex. If for all $x \in V(G), \operatorname{deg}(x)+\nu(G-$ $N[x]) \leqslant r$, then $e(G) \leqslant r^{2}$. Moreover, equality holds if and only if $G=K_{r, r}$.

Proof. Clearly, $\Delta(G) \leqslant r$. We claim that $\nu(G) \leqslant r$. Let $u_{1} v_{1}, \cdots, u_{\ell} v_{\ell}$ be a matching in $G$. Without loss of generality, assume that

$$
\begin{aligned}
& \left\{u_{1}, v_{1}, \cdots, u_{s}, v_{s}, u_{s+1}, \cdots, u_{s+t}\right\} \subseteq N\left[u_{1}\right], \\
& \left\{v_{s+1}, \cdots, v_{s+t}, u_{s+t+1}, v_{s+t+1}, u_{\ell}, v_{\ell}\right\} \subseteq V(G) \backslash N\left[u_{1}\right] .
\end{aligned}
$$


Then $s \geqslant 1,2 s+t-1 \leqslant \operatorname{deg}\left(u_{1}\right)$ and $\ell-(s+t) \leqslant \nu\left(G-N\left[u_{1}\right]\right)$. Thus,

$$
\ell \leqslant \nu\left(G-N\left[u_{1}\right]\right)+s+t \leqslant \operatorname{deg}\left(u_{1}\right)+\nu\left(G-N\left[u_{1}\right]\right) \leqslant r,
$$

the claim is true. Now we prove the result according to the following two cases.

Case 1. Suppose $\Delta(G)<r$. Then, by Lemma 3, we have $e(G) \leqslant f(\nu, \Delta) \leqslant r(r-1+$ 1) $=r^{2}$, and the equality holds only if $\nu=r$ and $\Delta=r-1$. We claim that the equality does not hold in this case. For $r \geqslant 4, e(G) \leqslant f(r, r-1)=r(r-1)+\left\lfloor\frac{r-1}{2}\right\rfloor\left\lfloor\frac{r}{\lceil(r-1) / 2\rceil}\right\rfloor<r^{2}$. For $r=3, e(G) \leqslant f(3,2)=3^{2}=9$. Suppose to the contrary that $e(G)=9$. Since $\Delta(G) \leqslant 2$ and $G$ has no isolated vertex, $|V(G)| \geqslant e(G)=9$ (equality holds if and only if $G$ is 2-regular) and $\omega(G) \leqslant \nu(G)=3$. By Observation 5 ,

$$
3=\nu(G) \geqslant \frac{|V(G)|-\omega(G)}{2} \geqslant \frac{|V(G)|-3}{2} .
$$

Hence $|V(G)| \geqslant 9$. Thus $|V(G)|=9$ (and so $G$ is 2-regular) and $\omega(G)=3$. Therefore, $G$ consists of three vertex-disjoint triangles. But this contradicts the assumption that $\operatorname{deg}(x)+\nu(G-N[x]) \leqslant r$ for all $x \in V(G)$.

Case 2. Suppose $\Delta(G)=r$. Choose $x \in V(G)$ such that $\operatorname{deg}(x)=r$, then $\nu(G-$ $N[x])=0$. Hence $e(G-N[x])=0$ and so each vertex in $G-N[x]$ must be adjacent to vertices in $N(x)$. Let $N(x)=\left\{x_{1}, \cdots, x_{r}\right\}$. For each $i \in[1, r]$, let $d_{i}=\operatorname{deg}\left(x_{i}\right)$ and $\tilde{d}_{i}=\operatorname{deg}_{G[N(x)]}\left(x_{i}\right)$. Then

$$
\begin{aligned}
e(G) & =e(G[N(x)])+e(N(x), V(G) \backslash N(x))=\frac{1}{2} \sum_{i=1}^{r} \tilde{d}_{i}+\sum_{i=1}^{r}\left(d_{i}-\tilde{d}_{i}\right) \\
& =\sum_{i=1}^{r} d_{i}-\frac{1}{2} \sum_{i=1}^{r} \tilde{d}_{i} \leqslant r^{2}-\frac{1}{2} \sum_{i=1}^{r} \tilde{d}_{i} \leqslant r^{2} .
\end{aligned}
$$

Moreover, equality holds if and only if $d_{i}=r$ and $\tilde{d}_{i}=0$ for each $i \in[1, r]$, that is, $G$ is a bipartite graph with partite sets $N(x)=\left\{x_{1}, \cdots, x_{r}\right\}$ and $V(G) \backslash N(x)$. To show that $G=K_{r, r}$, it suffices to prove that $|V(G) \backslash N(x)|=r$. If not, then $|V(G) \backslash N(x)|>r$. Since $\operatorname{deg}\left(x_{1}\right)=d_{1}=r$, there must exist a vertex $y \in(V(G) \backslash N(x)) \backslash N\left(x_{1}\right)$. Since $G$ has no isolated vertex, $y$ must be adjacent to some vertex $x_{j}$ with $j \neq 1$. This implies that $\nu\left(G-N\left[x_{1}\right]\right) \geqslant 1$, a contradiction with $\operatorname{deg}\left(x_{1}\right)+\nu\left(G-N\left[x_{1}\right]\right) \leqslant r$.

The following lemma states that the members $\mathcal{F}_{n, k}$ are $C_{k, q}$-free.

Lemma 7. Each member of $\mathcal{F}_{n, k}$ is $C_{k, q}$-free for all $k \geqslant 2, n \geqslant 4(k-1)^{2}$, and odd integer $q \geqslant 5$.

Proof. Suppose to the contrary that there is a graph $G \in \mathcal{F}_{n, k}$ containing a copy of $C_{k, q}$. Let $K$ be the copy of $K_{k-1, k-1}$ in $G$. Then each odd cycle of $C_{k, q}$ must contain odd number of the edges of $K$. Let $A=E\left(C_{k, q}\right) \cap E(K)$. Then $|A| \geqslant k$. We claim that the center of $C_{k, q}$ must lie in $K$. If not, then $G[A]$ contains a matching of order at least $k$ by the 
structure of $C_{k, q}$, a contradiction with $\nu(K)=k-1$. Let $x \in V(K)$ be the center of $C_{k, q}$. Assume that $\operatorname{deg}_{G[A]}(x)=s$ and let $E_{A}(x)$ be the set of edges incident with $x$ in $G[A]$. Then at most $s$ cycles of $C_{k, q}$ intersect $E_{A}(x)$, that is $A-E_{A}(x)$ contains a matching of $K$ of order at least $k-s$. This is impossible since $\nu\left(K-N_{G[A]}(x)\right) \leqslant k-s-1$.

Lemma 8. Let $n_{0}$ be an integer and let $G$ be a graph on $n \geqslant n_{0}+\left(\begin{array}{c}n_{0} \\ 2\end{array}\right)$ vertices with $e(G)=e\left(T_{n, 2}\right)+j$ for some integer $j>0$. Then $G$ contains a subgraph $G^{\prime}$ on $n^{\prime}>n_{0}$ vertices such that $\delta\left(G^{\prime}\right) \geqslant \delta\left(T_{n^{\prime}, 2}\right)$ and $e\left(G^{\prime}\right) \geqslant e\left(T_{n^{\prime}, 2}\right)+j+n-n^{\prime}$.

Proof. If $\delta(G) \geqslant\left\lfloor\frac{n}{2}\right\rfloor$, then $G$ is the desired graph and we have nothing to do. So assume that $\delta(G)<\left\lfloor\frac{n}{2}\right\rfloor$. Choose $v \in V(G)$ with $\operatorname{deg}_{G}(v)<\left\lfloor\frac{n}{2}\right\rfloor$. Let $G_{1}=G-v$. Then $e\left(G_{1}\right) \geqslant$ $e(G)-\operatorname{deg}_{G}(v) \geqslant e\left(T_{n, 2}\right)+j-\left\lfloor\frac{n}{2}\right\rfloor+1=e\left(T_{n-1,2}\right)+j+1$, since $e\left(T_{n, 2}\right)-e\left(T_{n-1,2}\right)=\left\lfloor\frac{n}{2}\right\rfloor$. We may continue this procedure until we get $G^{\prime}$ on $n-i$ vertices with $\delta\left(G^{\prime}\right) \geqslant \delta\left(T_{n-i, 2}\right)$ for some $i<n-n_{0}$, or until $i=n-n_{0}$. For the latter case, $G^{\prime}$ has $n_{0}$ vertices but $e\left(G^{\prime}\right) \geqslant e\left(T_{n_{0}, 2}\right)+j+i>n-n_{0} \geqslant\left(\begin{array}{c}n_{0} \\ 2\end{array}\right)$, which is impossible.

\section{Proof of Theorem 2}

Let $G$ be an extremal graph for $C_{k, q}$ on $n \geqslant n_{1}(k, q)\left(n_{1}(k, q)\right.$ is given below) vertices. By Lemma 7, $e(G) \geqslant e\left(T_{n, 2}\right)+(k-1)^{2}$. We will show that $e(G)=e\left(T_{n, 2}\right)+(k-1)^{2}$ and $G$ is a member of $\mathcal{F}_{n, k}$. Let

$$
\begin{aligned}
& \gamma=\frac{1}{1600}, \\
& n_{0}=n_{0}\left(C_{k, q}, \gamma\right) \quad\left(\text { which is determined by } C_{k, q} \text { and } \gamma \text { by applying Lemma } 4\right), \\
& n_{1}=n_{1}(k, q)=n_{0}+20 k^{2} q+\left(\begin{array}{c}
n_{0}+20 k^{2} q \\
2
\end{array}\right) .
\end{aligned}
$$

By the choice of $n_{1}$ and Lemma 8, we may assume $\delta(G) \geqslant \delta\left(T_{n, 2}\right)=\left\lfloor\frac{n}{2}\right\rfloor$, otherwise, we consider a subgraph $G^{\prime}$ with the desired minimum degree instead of $G$. Let $V_{0} \dot{\cup} V_{1}$ be a partition of $V(G)$ such that $e\left(V_{0}, V_{1}\right)$ is maximized. Lemma 4 implies that $m=$ $e\left(V_{0}\right)+e\left(V_{1}\right)<\gamma n^{2}$. The following claim asserts that the partition is close to being balanced. The following inequality holds:

\section{Claim 9.}

$$
\frac{n}{2}-\sqrt{\gamma} n<\left|V_{i}\right|<\frac{n}{2}+\sqrt{\gamma} n \text { for } i=0,1 .
$$

Furthermore, $m=e\left(V_{0}\right)+e\left(V_{1}\right) \geqslant(k-1)^{2}$ and if the equality holds then $G$ contains a complete balanced bipartite graph with classes $V_{0}$ and $V_{1}$.

Proof. Let $\left|V_{0}\right|=\frac{n}{2}+a$. Then $\left|V_{1}\right|=\frac{n}{2}-a$. Since

$$
\left\lfloor\frac{n^{2}}{4}\right\rfloor+(k-1)^{2}=e\left(T_{n, 2}\right)+(k-1)^{2} \leqslant e(G) \leqslant\left|V_{0}\right|\left|V_{1}\right|+m=\frac{n^{2}}{4}-a^{2}+m,
$$

we have $m \geqslant(k-1)^{2}$ and $m \geqslant a^{2}$. Since $m<\gamma n^{2}, a^{2}<\gamma n^{2}$. Hence $|a|<\sqrt{\gamma} n$. 
If $m=(k-1)^{2}$, then

$$
e\left(T_{n, 2}\right)+(k-1)^{2} \leqslant e(G)=e\left(V_{0}, V_{1}\right)+(k-1)^{2} .
$$

Hence $e\left(V_{0}, V_{1}\right)=e\left(T_{n, 2}\right)$, that is, $V_{0}, V_{1}$ are balanced and so $G$ contains a complete balanced bipartite graph with classes $V_{0}$ and $V_{1}$.

In the following, let $G_{i}=G\left[V_{i}\right], \Delta_{i}=\Delta\left(G_{i}\right)$ and $\nu_{i}=\nu\left(G_{i}\right), i=0,1$. For a vertex $x \in V_{i}$, let $E_{1-i}(x)=\left\{e \in E\left(G_{1-i}\right) \mid V(e) \cap N_{G}(x) \neq \emptyset\right\}$.

Claim 10. For any vertex $x \in V_{i}$,

$$
\operatorname{deg}_{G_{i}}(x)+\nu\left(G_{i}-N_{G_{i}}[x]\right)+\nu\left(G\left[E_{1-i}(x)\right]\right) \leqslant k-1 .
$$

Proof. We prove this claim by contradiction. Without loss of generality, assume that there is an $x \in V_{0}$ such that $\operatorname{deg}_{G_{0}}(x)+\nu\left(G_{0}-N_{0}[x]\right)+\nu\left(G\left[E_{1}(x)\right]\right) \geqslant k$. Let $x x_{1}, x x_{2}, \ldots, x x_{s} \in$ $E\left(G_{0}\right)$, and let $M_{0}=\left\{u_{s+1} v_{s+1}, \ldots u_{t} v_{t}\right\}$ be a matching of $G\left[V_{0} \backslash\left\{x, x_{1}, \ldots, x_{s}\right\}\right]$ and $M_{1}=\left\{u_{t+1} v_{t+1}, \ldots, u_{k} v_{k}\right\}$ a matching of $G\left[E_{1}(x)\right]$ such that $x u_{t+1}, \ldots, x u_{k} \in E_{G}\left(x, V_{1}\right)$, where $s, t \in[0, k]$. Define

$$
\xi(j)= \begin{cases}1 & \text { if } j \text { is odd } \\ 0 & \text { if } j \text { is even }\end{cases}
$$

We say that $v$ is bad if $\operatorname{deg}_{G\left[V_{i}\right]}(v)>t_{1}$, otherwise $v$ is said to be $\operatorname{good}$, where $t_{1}=6 \sqrt{\gamma} n$. Then the number of bad vertices in $G$ is at most $\frac{2 m}{t_{1}}<\frac{2 \gamma n^{2}}{t_{1}}=\frac{1}{3} \sqrt{\gamma} n$.

For any vertex $u \in V_{i}(i=0,1)$, by the maximality of $e\left(V_{0}, V_{1}\right)$, we have

$$
e_{G}\left(u, V_{1-i}\right) \geqslant \max \left\{e_{G}\left(u, V_{i}\right),\left\lfloor\frac{n}{2}\right\rfloor-e_{G}\left(u, V_{i}\right)\right\} \geqslant \frac{1}{2}\left\lfloor\frac{n}{2}\right\rfloor .
$$

Particularly, if $u \in V_{i}$ is good, then we have

$$
e_{G}\left(u, V_{1-i}\right) \geqslant\left\lfloor\frac{n}{2}\right\rfloor-e_{G}\left(u, V_{i}\right) \geqslant\left\lfloor\frac{n}{2}\right\rfloor-t_{1}\left(\geqslant \frac{1}{2}\left\lfloor\frac{n}{2}\right\rfloor\right) .
$$

Let $A=V\left(\left\{x x_{1}, x x_{2}, \ldots, x x_{s}\right\} \cup M_{i} \cup M_{1-i}\right)$. We find a copy of $C_{k, q}$ passing through all the vertices of $A$ to get a contradiction.

For each $\ell \in[1, s]$, we find a sequence of vertices $w_{1 \ell}^{1}, w_{0 \ell}^{2}, \cdots, w_{0 \ell}^{q-3}, w_{1 \ell}^{q-2}$ with $w_{\xi(j) \ell}^{j} \in$ $V_{\xi(j)} \backslash A$ for $1 \leqslant j \leqslant q-2$, such that $w_{0 \ell}^{q-3}$ is good and $x w_{1 \ell}^{1} \cdots w_{0 \ell}^{q-3} w_{1 \ell}^{q-2} x_{\ell} x$ is a $q$-cycle. Furthermore, we require that $w_{\xi(j) \ell}^{j}(\ell \in[1, s], j \in[1, q-2])$ are pairwise different. This is possible since together with all vertices in $A$, the total number of good vertices which we have found is at most $\left|V\left(C_{k, q}\right)\right|=k(q-1)+1$ and each vertex $u \in V_{i}$ has at least

$$
e_{G}\left(u, V_{1-i}\right)-\frac{1}{3} \sqrt{\gamma} n \geqslant \frac{1}{2}\left\lfloor\frac{n}{2}\right\rfloor-\frac{1}{3} \sqrt{\gamma} n \geqslant\left|V\left(C_{k, q}\right)\right|\left(\text { since } n \geqslant 20 k^{2} q\right)
$$

good neighbors in $V_{1-i}$ and the number of common neighbors of $w_{0 \ell}^{q-3}$ and $x_{\ell}$ in $V_{1}$ is at least (since $n \geqslant 20 k^{2} q$ )

$$
e_{G}\left(w_{0 \ell}^{q-3}, V_{1}\right)+e_{G}\left(x_{\ell}, V_{1}\right)-\left|V_{1}\right| \geqslant\left\lfloor\frac{n}{2}\right\rfloor-t_{1}+\frac{1}{2}\left\lfloor\frac{n}{2}\right\rfloor-\left(\frac{n}{2}+\sqrt{\gamma} n\right) \geqslant\left|V\left(C_{k, q}\right)\right| .
$$


Thus we have found a copy of $C_{s, q}$ centered at $x$ and passing through the edges of $\left\{x x_{1}, x x_{2}, \cdots, x x_{s}\right\}$. Particularly, since $G$ is $C_{k, q}$-free, we have $s \leqslant k-1$. Thus

$$
\Delta_{i}=\Delta\left(G_{i}\right) \leqslant k-1<t_{1}, i=0,1 .
$$

Consequently, all the vertices of $G$ are good, and for each vertex $u \in V_{i}$,

$$
e_{G}\left(u, V_{1-i}\right) \geqslant\left\lfloor\frac{n}{2}\right\rfloor-(k-1) \geqslant \frac{n}{2}-k
$$

and thereby

$$
\frac{n}{2}-k \leqslant\left|V_{i}\right| \leqslant \frac{n}{2}+k, i=0,1
$$

Next we will find a copy of $C_{k-s, q}$ centered at $x$ disjoint from the copy of $C_{s, q}$. For every $u_{\ell}(\ell \in[s+1, t])$, choose a common neighbor of $x$ and $u_{l}$, say $w_{1 \ell}^{1}$, in $V_{1}$ such that $w_{1 \ell}^{1} \neq w_{1 \ell^{\prime}}^{1}$ if $\ell \neq \ell^{\prime}$. We can do this because the number of common neighbors of $x$ and $u_{\ell}$ in $V_{1}$ is at least

$$
e_{G}\left(x, V_{1}\right)+e_{G}\left(u_{\ell}, V_{1}\right)-\left|V_{1}\right| \geqslant 2\left(\frac{n}{2}-k\right)-\left(\frac{n}{2}+k\right) \geqslant\left|V\left(C_{k, q}\right)\right|\left(\text { since } n \geqslant 20 k^{2} q\right) .
$$

For each $\ell \in[s+1, t]$, with the same reason as above, we find vertices $w_{1 \ell}^{3}, w_{0 \ell}^{4}, \cdots, w_{0 \ell}^{q-3}$ one by one, then a common neighbor of $w_{0 \ell}^{q-3}$ and $v_{\ell}$, say $w_{1 \ell}^{q-2}$, in $V_{1}$ such that

$$
u_{\ell} w_{1 \ell}^{1} x w_{1 \ell}^{3} \cdots w_{1 \ell}^{q-2} v_{\ell} u_{\ell}
$$

is a $q$-cycle. And for every $l \in[t, k]$, begin with $x$, we can find vertices $w_{1 \ell}^{2}, w_{0 \ell}^{3}, \cdots, w_{1 \ell}^{q-3}$ one by one, then a common neighbor, say $w_{0 \ell}^{q-2}$, of $w_{1 \ell}^{q-3}$ and $v_{\ell}$ in $V_{0}$ such that

$$
u_{\ell} x w_{1 \ell}^{2} w_{0 \ell}^{3} \cdots w_{0 \ell}^{q-2} v_{\ell} u_{\ell}
$$

is a $q$-cycle. Also, we may require that $w_{\xi(j) \ell}^{j}(\ell \in[s+1, k], j \in[1, q-2])$ are pairwise different. Thus the $k-s q$-cycles form a copy of $C_{k-s, q}$ centered at $x$ and passing through all of the edges of $M_{0} \cup M_{1}$. Therefore, $G\left[\left\{x, x_{1}, \cdots, x_{l}\right\} \cup\left\{w_{\xi(j), l}^{j}: j \in[1, q-2], \ell \in[1, k]\right\}\right]$ contains a desired copy of $C_{k, q}$ with center $x$. This completes the proof.

Claim 11. We have that $\nu_{0}+\nu_{1} \leqslant k-1$.

Proof. If not, suppose that $M_{0}=\left\{u_{1} v_{1}, \cdots, u_{s} v_{s}\right\}$ and $M_{1}=\left\{u_{s+1} v_{s+1}, \cdots, u_{k} v_{k}\right\}$ are matchings in $G_{0}$ and $G_{1}$, respectively. Without loss of generality, assume that $s \geqslant 1$. First we find a common neighbor, say $x$, of $u_{s+1}, \cdots, u_{k}$ in $V_{0} \backslash V\left(M_{0}\right)$. This is possible since the number of such neighbors is at least

$$
\begin{aligned}
& \sum_{l=s+1}^{k} e_{G}\left(u_{\ell}, V_{0}\right)-(k-s-1)\left|V_{0}\right|-2 s \\
\geqslant & (k-s)\left(\frac{n}{2}-k\right)-(k-s-1)\left(\frac{n}{2}+k\right)-2 k \\
= & \frac{n}{2}-(2(k-s)+1) k \\
\geqslant & \frac{n}{2}-2 k^{2}>0\left(\text { since } n \geqslant 20 k^{2} q\right) .
\end{aligned}
$$


Let $M_{0}^{\prime}$ be the maximal subset of $M_{0}$ such that $M_{0}^{\prime}$ is a matching of $G_{0}-N_{G_{0}}[x]$. By Claim 10, $\operatorname{deg}_{G_{0}}(x)+\left|M_{0}^{\prime}\right|+\left|M_{1}\right| \leqslant k-1$. Clearly, $\operatorname{deg}_{G_{0}}(x)+\left|M_{0}^{\prime}\right| \geqslant s$. Hence $\operatorname{deg}_{G_{0}}(x)+$ $\left|M_{0}^{\prime}\right|+\left|M_{1}\right| \geqslant k$, a contradiction.

Claim 12. We have that $\max \left\{\Delta_{0}, \Delta_{1}\right\}=k-1$.

Proof. If not, then by Claim $10, \max \left\{\Delta_{0}, \Delta_{1}\right\} \leqslant k-2$. Thus by Lemma 3 and Claim 11,

$$
\begin{aligned}
m & =e\left(V_{0}\right)+e\left(V_{1}\right) \leqslant f\left(\nu_{0}, k-2\right)+f\left(\nu_{1}, k-2\right) \\
& \leqslant f\left(\nu_{0}+\nu_{1}, k-2\right) \leqslant f(k-1, k-2) .
\end{aligned}
$$

If $k \neq 4, m \leqslant f(k-1, k-2)=(k-1)^{2}-1$, contradicts to $m \geqslant(k-1)^{2}$ (by Claim 9).

If $k=4$, then $m \leqslant f(3,2)=(k-1)^{2}=9$. By Claim $9, m=(k-1)^{2}=9$ and $G$ contains a complete balanced bipartite subgraph with classes $V_{0}$ and $V_{1}$. Let $H$ be the subgraph consisting of nonempty components of $G_{0} \cup G_{1}$. Then $H$ is a graph with $e(H)=9, \Delta(H)=2$ and $\nu(H)=3$. By Observation 5 and with a similar discussion as in Case 1 of the proof of Lemma $6, H$ consists of three vertex-disjoint triangles. Then we can easily find a vertex $x$ in $H$ with $\operatorname{deg}_{H}(x)=2$ and a matching of order 2 in $H-N_{H}[x]$. That is $\operatorname{deg}_{G_{i}}(x)+\nu\left(G_{i}-N_{G_{i}}[x]\right)+\nu\left(G\left[E_{1-i}(x)\right]\right) \geqslant 4=k$, a contradiction Claim 10 .

Claim 13. We have that $e\left(V_{0}\right) \cdot e\left(V_{1}\right)=0$.

Proof. At first, by Claim 12 and Claim 11, we have

$$
\begin{aligned}
m & \leqslant f\left(\nu_{0}, k-1\right)+f\left(\nu_{1}, k-1\right) \leqslant f\left(\nu_{0}+\nu_{1}, k-1\right) \\
& \leqslant f(k-1, k-1) \leqslant k(k-1) .
\end{aligned}
$$

Next, by Claim 12, $\max \left\{\Delta_{0}, \Delta_{1}\right\}=k-1$. Without loss of generality, assume $\Delta_{0}=$ $k-1$. Let $x \in V_{0}$ with $\operatorname{deg}_{G_{0}}(x)=k-1$. We show that $e\left(V_{1}\right)=0$. If $e\left(V_{1}\right)>0$, then $\nu_{1} \geqslant 1$. By Claim 11, $\nu_{0} \leqslant k-1-\nu_{1}=k-2$. Let $A_{1}=\left\{u \in V_{1}: \operatorname{deg}_{G_{1}}(u)>0\right\}$. By Claim 10, we have $A_{1} \cap N_{G}(x)=\emptyset$. So $e\left(V_{0}, V_{1}\right) \leqslant\left|V_{0}\right|\left|V_{1}\right|-\left|A_{1}\right| \leqslant e\left(T_{n, 2}\right)-\left|A_{1}\right|$. Thus we have

$$
e\left(T_{n, 2}\right)+(k-1)^{2} \leqslant e(G) \leqslant e\left(T_{n, 2}\right)-\left|A_{1}\right|+m .
$$

Therefore, $\left|A_{1}\right| \leqslant m-(k-1)^{2}(\leqslant k-1)$. Again by Lemma 3, we have

$$
\begin{aligned}
m & \leqslant \nu_{0}\left(\Delta_{0}+1\right)+\nu_{1}\left(\Delta_{1}+1\right) \\
& \leqslant k \nu_{0}+\left(k-1-\nu_{0}\right)\left|A_{1}\right|\left(\text { since } \Delta_{1}+1 \leqslant\left|A_{1}\right|\right) \\
& =\nu_{0}\left(k-\left|A_{1}\right|\right)+(k-1)\left|A_{1}\right| \\
& \leqslant(k-2)\left(k-\left|A_{1}\right|\right)+(k-1)\left|A_{1}\right|\left(\text { since }\left|A_{1}\right| \leqslant k-1 \text { and } \nu_{0} \leqslant k-2\right) \\
& =(k-1)^{2}+\left|A_{1}\right|-1 \leqslant(k-1)^{2}+m-(k-1)^{2}-1 \\
& =m-1, \text { a contradiction. }
\end{aligned}
$$

By Claim 13, Without loss of generality, we may assume $e\left(V_{1}\right)=0$, so $m=e\left(V_{0}\right)$. Let $A_{0}$ be the set of non-isolated vertices in $G_{0}$. By Claim 10 and Lemma 6, $m=e\left(G\left[A_{0}\right]\right) \leqslant$ $(k-1)^{2}$. By Claim 9, we must have $m=(k-1)^{2}$ and therefore $G$ contains a complete balanced bipartite subgraph with classes $V_{0}$ and $V_{1}$. Again by Claim 10 and Lemma 6, since $e\left(G\left[A_{0}\right]\right)=(k-1)^{2}, G\left[A_{0}\right]$ must be a copy of $K_{k-1, k-1}$. This completes the proof of Theorem 2. 


\section{References}

[1] V. Chavátal, D. Hanson, Degrees and matchings, J. Combin. Theory Ser. B, 20(2): 128-138, 1976.

[2] G. Chen, R. J. Gould, F. Pfender, and B. Wei, Extremal graphs for intersecting cliques, J. Combin. Theory Ser. B 89: 159-171, 2003.

[3] P. Erdős, On some new inequalities concerning extremal properties of graphs, In Theory of Graphs, Proc. Colloq., Tihany, 1966, Academic Press, New York, pp. 77-81, 1968.

[4] P. Erdős, Z. Füredi, R. J. Gould, D. S. Gunderson, Extremal graphs for intersecting triangles, J. Combin. Theory Ser. B, 64(1): 89-100, 1995.

[5] R. Glebov, Extremal graphs for clique-paths, arXiv:1111.7029v1.

[6] H. Liu, Extremal graphs for blow-ups of cycles and trees, The Electron. J. of Combin., 20(1): P65, 2013.

[7] M. Simonovits, A method for solving extremal problems in graph theory, stability problems, In Theory of Graphs, Proc. Colloq., Tihany, 1966, Academic Press, New York, pp. 279-319, 1968. 